\title{
An Autocorrelation Modeling Method for Oxygen Saturation Measurement During Low Perfusion
}

\section{Shuangping Tan ( $\nabla$ tanshuangpingzy@163.com )}

The Jiangxia District No.1 People's Hospital of Wuhan, Wuhan, 430200

\section{Jie Wei}

The Jiangxia District No.1 People's Hospital of Wuhan, Wuhan, 430200

\section{Hao Chen}

The Jiangxia District No.1 People's Hospital of Wuhan, Wuhan, 430200

\section{Tong Zhang}

State Key Laboratory of Information Engineering in Surveying, Mapping and Remote Sensing, Wuhan University, Wuhan, 430079

\section{Xiali Wu}

The Jiangxia District No.1 People's Hospital of Wuhan, Wuhan, 430200

\section{Youfeng Deng}

The Jiangxia District No.1 People's Hospital of Wuhan, Wuhan, 430200 Hongbin Zuo

The Jiangxia District No.1 People's Hospital of Wuhan, Wuhan, 430200

\section{Research Article}

Keywords: Pulse oximetry, SpO2 extraction, Low perfusion, Autocorrelation modeling method

Posted Date: January 23rd, 2021

DOI: https://doi.org/10.21203/rs.3.rs-150041/v1

License: (c) (1) This work is licensed under a Creative Commons Attribution 4.0 International License.

Read Full License 


\section{An autocorrelation modeling method for oxygen}

2 saturation measurement during low perfusion

3 Tan Shuangping**1, Wei Jie* ${ }^{* 1}$, Chen $\mathrm{Hao}^{* 1}{ }^{1}$, Zhang Tong ${ }^{2}$, Wu Xiali ${ }^{1}$, Deng Youfeng ${ }^{1}$,

4 Zuo Hongbin ${ }^{\# 1}$

$5 *$ These authors contribute equally to this work

$6 \quad$ \# Correspondence: tanshuangpingzy@163.com

7 Affiliation:

8 1. The Jiangxia District No.1 People's Hospital of Wuhan, Wuhan, 430200, China

9 2. State Key Laboratory of Information Engineering in Surveying, Mapping and Remote

10 Sensing, Wuhan University, Wuhan, 430079, China

12 Abstract:

13 Background: $\mathrm{SpO}_{2}$ is a widely used estimation of oxygen saturation owing to its

14 convenient usage and low cost. However, $\mathrm{SpO}_{2}$ determination under low perfusion

15 condition is severely affected by noise.

16 Methods: In this paper, an autocorrelation modeling method for the oxygen saturation

17 measurement during low perfusion is presented. The proposed method mainly contains

18 two steps: calculating the autocorrelation of the photoplethysmography (PPG) signals and

19 modeling for the parameter calculation. The autocorrelation of the PPG signals can

20 suppress the noise and extract pulse waves from low perfusion signals. The model can

21 realize the calculation of $\mathrm{SpO}_{2}$ with the autocorrelation signals.

22 Results: Experiments showed that the new method had a good accuracy and stability

23 under low perfusion condition (perfusion index $(\mathrm{PI})<=0.2 \%$ ), and it was also motion- 

saturation measurement under the condition of lower perfusion( $\mathrm{PI}=0.1 \%)$.

tolerant. Meanwhile, the new method showed a good performance for the oxygen

Conclusions: The new method could realize the calculation of $\mathrm{SpO} 2$ by little computation and high efficiency without extra hardware. It has strong potential in real-time clinical use.

Keywords: Pulse oximetry, $\mathrm{SpO} 2$ extraction, Low perfusion, Autocorrelation modeling method

\section{Background}

Saturation of peripheral oxygen $\left(\mathrm{SpO}_{2}\right)$, which reflects arterial oxygenation levels, is often used to assess the function of the respiratory system and the circulation system. Pulse oximetry is a non-invasive technique, recording from extremities such as fingers and ear lobes, to continuously monitor $\mathrm{SpO}_{2}$. Owing to its cost-effectiveness benefits, pulse oximetry has become a regular monitoring device used in anesthesia, surgical operation, and intensive care ${ }^{[1,2]}$. However, clinical applications of pulse oximetry revealed that the measurement accuracy and precision were severely affected by motion and low peripheral perfusion caused by low ambient temperature or cardiogenic shock ${ }^{[3,4]}$. In case of low perfusion, the amplitude of photoplethysmography (PPG) signals, which is used to calculate $\mathrm{SpO}_{2}$, become very small and hidden in noise. The signal-to-noise ratio (SNR) is too low to extract useful information with conventional methods(Fig. $1 \mathrm{~b}$ ). In case of motion, PPG signals are contaminated by motion artifacts. The frequency spectrum overlap of PPG signals with motion artifacts makes conventional low-pass or high-pass filter techniques inapplicable. Therefore, new methods are needed to deal with the issue mentioned above. 
To overcome the shortcomings of motion and low perfusion, many algorithms were developed in the past few years. For instance, Masimo Corporation proposed Discrete Saturation Transform (DST) method to adaptively filter out noise from recordings by constructing a reference noise ${ }^{[5] .}$ Yan et al. proposed a robust minimum correlation discrete saturation transform (MCDST) algorithm to remove motion artifact ${ }^{[6]}$. Yousefi et al. developed a real-time adaptive algorithm to extract heart rate and $\mathrm{SpO}_{2}$ for a wireless pulse oximeter allowing users to move freely ${ }^{[7]}$. Byung et al. proposed independent component analysis to reduce noise in PPG signals ${ }^{[8]}$. However, this method did not perform well for low perfusion interference. Foo proposed two algorithms to account for the difference between the properties of low perfusion signal and motion contaminated PPG ${ }^{[9]}$. However, Foo's method for low perfusion case was a complicated non-causal filter technique commonly used for off-line analysis. In addition, $\mathrm{SpO}_{2}$ was not extracted in their work. Therefore, new algorithms for accurately extracting $\mathrm{SpO}_{2}$ under low perfusion is required, especially for the anesthesia, surgical operation, and intensive care application. low perfusion conditions. First, we characterize the properties of PPG (photoplethysmography) signals under low perfusion condition. Then, we deduce the new autocorrelation based $\mathrm{SpO}_{2}$ extraction method. At last, validation test resutls of the method on accuracy, stability, and motion-tolerance are presented. The new $\mathrm{SpO}_{2}$ extraction method have potential of real-time clinical use.

\section{Methods}

To realize the accurate measurement of $\mathrm{SpO}_{2}$ under low perfusion condition, we

71 designed a pulse oximetry to acquire PPG signals(generated by a $\mathrm{SpO}_{2}$ simulator-Fluke Index 2XL) which are pre-processed and used for calculating the alternative 
component(AC) and the direct component(DC). We computed autocorrelation on recorded AC signals, then calculated the $\mathrm{SpO}_{2}$ with the autocorrelation preprocessed signals by our modeled method. The overall process is shown in Fig. 2.

\subsection{PPG signal generation}

Photoplethysmography (PPG) signals, which are used to extract $\mathrm{SpO}_{2}$, can be obtained when illuminating the body with a light beam at a haemoglobin-sensitive wavelength. In the peripheral sites to be recorded, the volume of blood within the illuminating field alters periodically to the artery pulsation, thus generating fluctuating PPG signals. A PPG signal can be represented as an addition of an alternative component (AC) and a direct component (DC). The DC signals reflect lights that are absorbed or scattered by vein, base volume of artery, and other non-pulsatile tissue, while the AC signals reflect volume change in the artery and arterial blood. The parameter PI (perfusion index) is an indication of the peripheral perfusion level, which can be simply calculated by the ratio of AC to DC amplitude $\left(\mathrm{PI}=\mathrm{P}_{\mathrm{AC}} / \mathrm{P}_{\mathrm{DC}}\right)$. In the low perfusion case, $\mathrm{PI}$ can be less than $0.2 \%$.

To develop the new method, two kinds of PPG signals were used: 1) simulated signals, and 2) recorded signals. A way to model low perfusion condition is lowering the peripheral temperature. However, its controllability and reproducibility are relatively low. Therefore, we used simulated PPG signals generated by a $\mathrm{SpO}_{2}$ simulator-Fluke Index 2XL (Fluke Biomedical, USA). With the simulator, we could obtain PPG signals with preset PI2 (PI for infrared case), $\mathrm{PR}$ (pulse rate), and $\mathrm{SpO}_{2}$ (Fig. 1). Signal simulation is a good tool for developing and testing the new method for low perfusion case. On the other hand, motion artifacts were modeled by direct recording the PPG as the users moved their fingers. A home-made reusable dual-wavelength PPG sensors were used in this case. 


\subsection{Calculation of $\mathrm{SpO}_{2}$}

97

98

99

100

101

102

103

$$
S p O_{2}=\frac{\varepsilon_{H b}^{\lambda_{2}} R-\varepsilon_{H b}^{\lambda_{1}}}{\left(\varepsilon_{H b O_{2}}^{\lambda_{1}}-\varepsilon_{H b}^{\lambda_{1}}\right)-\left(\varepsilon_{H b O_{2}}^{\lambda_{2}}-\varepsilon_{H b}^{\lambda_{2}}\right) R}
$$

105 Where, $\lambda_{1}$ and $\lambda_{2}$ are the two wavelengths (in 660 and $940 \mathrm{~nm}$ ); $R$ is the ratio of the two

106 PIs (i.e., $\left.R=\mathrm{PI}_{1} / \mathrm{PI}_{2}\right) ; \varepsilon_{H b O_{2}}^{\lambda_{1}}$ and $\varepsilon_{H b O_{2}}^{\lambda_{2}}$ are the extinction coefficients of $\mathrm{HbO}_{2}$ at $\lambda_{1}$ and

$107 \lambda_{2} ; \varepsilon_{H b}^{\lambda_{1}}$ and $\varepsilon_{H b}^{\lambda_{2}}$ are the extinction coefficients of $\mathrm{Hb}$ at $\lambda_{1}$ and $\lambda_{2}$. All the relevant

108 coefficients are constant ${ }^{[10]}$. Therefore, $\mathrm{SpO}_{2}$ is determined as $R$ is obtained from

109 recordings. Considering individual differences in scattering effect and other uncertainty

110 factors, Eq.1 is generally expanded in terms of second-order Taylor series in practice as

111 follows:

$$
\mathrm{SpO}_{2}=A^{*} R^{2}+B^{*} R+C
$$

113 Where the coefficients $A, B$, and $C$ can be calibrated by fitting the data to a conic curve 114 using Least Squares. 
116 Autocorrelation modeling consists of 1) autocorrelation function computation, and 2)

117 relating $\mathrm{SpO}_{2}$ and other parameters to autocorrelation signals. Before going into the

118 autocorrelation modeling details, we review the autocorrelation technique first.

119 Autocorrelation technique is a weak signal detection technique, particularly for signals

120 with cyclic properties, such as ECG. For a signal $x(t)$, and its autocorrelation function is

121 defined as

$$
R_{x}(\tau)=\lim _{T \rightarrow \infty} \frac{1}{T} \int_{0}^{T} x(t) x(t+\tau) \mathrm{d} t
$$

123 Now, we present a simple demonstration of noise reduction of autocorrelation

124 technique. Given a sinusoidal signal $s(t)=A \sin (\omega t+\varphi)$ and its noise $n(t)$, the signal $x(t)$ is a 125 sum of these two signals; i.e., $x(t)=s(t)+n(t)=A \sin (\omega t+\varphi)+n(t)$. Then, the autocorrelation 126 function of this signal is

$$
\begin{aligned}
& R_{x}(\tau)=\lim _{T \rightarrow \infty} \int_{0}^{T}[s(t)+n(t)][s(t+\tau)+n(t+\tau)] \mathrm{d} t \\
& =\lim _{T \rightarrow \infty} \int_{0}^{T} s(t) s(t+\tau) \mathrm{d} t+\lim _{T \rightarrow \infty} \int_{0}^{T} s(t) n(t+\tau) \mathrm{d} t \\
& \quad+\lim _{T \rightarrow \infty} \int_{0}^{T} n(t) s(t+\tau) \mathrm{d} t+\lim _{T \rightarrow \infty} \int_{0}^{T} n(t) n(t+\tau) \mathrm{d} t \\
& =R_{s}(\tau)+R_{s n}(\tau)+R_{n s}(\tau)+R_{n}(\tau)
\end{aligned}
$$

128 Since the original signal $s(t)$ and its noise $n(t)$ are independent, and if the noise is white

129 noise, the three latter terms tend to be zero as $\tau \rightarrow \infty$. Thus we have

$$
R_{x}(\tau)=R_{s}(\tau)=\frac{A^{2}}{2} \cos \omega \tau
$$

131 The autocorrelation function has the same frequency to the original signal, and the

132 amplitude can be related to that of the original. Therefore, useful information can be

133 extracted while noises are reduced ${ }^{[11,12]}$. 


\subsubsection{Autocorrelation modeling of $\mathrm{SpO}_{2}$}

PPG signals, are regenerative and can be regarded as cyclic in a small period of time.

Suppose the resolved AC component of PPG is denoted by $p_{A C}(t)$, then we have

$$
p_{A C}(t)=p_{A C}(t+m T)=P_{A C} \cdot g_{A C}(t)
$$

Where $T$ is the period of $p_{A C}(t) ; m$ is an arbitrary constant; $g_{A C}(t)$ is normalized $p_{A C}(t)$, and $P_{A C}$ is the amplitude of $p_{A C}(t)$. Recall that $\mathrm{SpO}_{2}$ is calculated from $R$, which is the ratio of the two PIs. Since PI is defined as the ratio of AC and DC amplitude, conventional computation of $R$ is as follows:

$$
R=\frac{P I_{1}}{P I_{2}}=\frac{P_{A C 1} / P_{D C 1}}{P_{A C 2} / P_{D C 2}}=\frac{P_{A C 1} / P_{A C 2}}{P_{D C 1} / P_{D C 2}}
$$

However, in the low perfusion case, both $P_{A C 1}$ and $P_{A C 2}$ are very weak and prone to be distorted by noise and interference, while the amplitude of DC component was less affected. To suppress noise, we compute autocorrelations on recorded AC signals. Suppose the noise in the recorded AC signal $p r_{A C}(t)$ is denoted by $n(t)$; i.e., $p r_{A C}(t)=p_{A C}$ $(t)+n(t)$. The autocorrelation function of $p r_{A C}(t)$ can be represent as follows:

$$
\begin{gathered}
R_{p r A C}(\tau)=R_{p A C}(\tau)+R_{p A C n}(\tau)+R_{n p A C}(\tau)+R_{n}(\tau) \\
=R_{p A C n}(\tau)+R_{n}(\tau)=P_{A C}^{\prime} \cdot g_{A C}^{\prime}(\tau)
\end{gathered}
$$

Where $R_{p A C n}(\tau)$ and $R_{n p A C}(\tau)$ are cross-correlations of $p_{A C}(t)$ and $n(t)$, which equal to zero as they are independent with each other. $g_{A C}^{\prime}(\tau)$ is normalized autocorrelation signals of $p r_{A C}(t)$ and $P_{A C}^{\prime}$ is the amplitude of autocorrelation signals of $p r_{A C}(t) . R_{n}(\tau)$ is only nonzero at $\tau=0$. Therefore, the weak cyclic pulsation signal was retrieved from the stable autocorrelation function with a strongly suppressed noise. Besides, since autocorrelation does not change the periodical property of the original signal, $R_{p r a C}(\tau)$ is also regenerative and has a period of $T$ and has an amplitude proportional to the square of $P_{A C}$; i.e., 


$$
P_{A C}=k \sqrt{P_{A C}^{\prime}}
$$

157 Where $k$ is the amplitude transfer factor (Fig. 3). Unlike sinusoidal signal, $k$ does not

158 exactly equal to $2^{1 / 2}$ and, in practice, we determined it empirically through experimental

159 trials. In this way, the amplitude of AC component of the PPG signal, i.e. $P_{A C}$, can be

160 determined.

161 Given $P_{A C}$, the perfusion index (PI) can be calculated from the amplitude of DC

162 component $\left(P_{D C}\right)$, which is relative easy to be determined; thus we have

$$
P I=P_{A C} / P_{D C}=\left(k \sqrt{P_{A C}^{\prime}}\right) / P_{D C}
$$

164 In the dual-wavelength method, we actually obtained two PIs corresponding to

165 wavelength $\lambda_{1}$ and $\lambda_{2}$, respectively. Therefore, with autocorrelation modeling method, we 166 obtained $R$ (denoted as $R$ ' to be distinguished from $R$ calculated with Eq. 7) as follows:

$$
R^{\prime}=\frac{P I_{1}}{P I_{2}}=\frac{k_{1} \sqrt{P_{A C 1}^{\prime}} / k_{2} \sqrt{P_{A C 2}^{\prime}}}{P_{D C 1} / P_{D C 2}}
$$

168 Where $k_{1}$ and $k_{2}$ are amplitude transfer factors at the two wavelengths. Thus we compute

$169 \mathrm{SpO}_{2}$ as follows:

$$
\mathrm{SpO}_{2}=A^{*} R^{\prime 2}+B * R^{\prime}+C
$$

\subsection{Implementation of $\mathrm{SpO}_{2}$}

172 To extract $\mathrm{SpO}_{2}$ accurately, the autocorrelation modeling technique, in practice, is comprised of four steps described as follows.

(1) Signal recording and pre-processing. The dual-wavelength signals were recorded and packed in a crosswise format for the convenience of data transmission. In the preprocessing step, data from the same source would be accumulated in a dedicated data pool 
177 for later use. After data-rearrangement, the raw data were low-pass filtered to reduce

178 noise, notch filtered to suppress power-line interference.

179 (2) $P_{A C}$ and $P_{D C}$ computation. As for $P_{D C}$, the computation was relatively simple: low-

180 pass filtering the raw data and calculating the mean value. As for $P_{A C}$, we high-pass

181 filtered the original PPG AC signal and computed the difference between peak and valley.

182 (3) Computation of autocorrelation function and its amplitude. In practice, the

183 summation limit to calculate autocorrelation function can never be infinite and should be

184 truncated:

$$
R_{x}(m)=\frac{1}{N} \sum_{n=0}^{N-1 \dashv|m|} x(n) x(n+m)
$$

186 Where, $N \gg>m$. The fast computation of autocorrelation is generally achieved by FFT and

187 window technique. To make the method be adaptive to real-time use, we employed the

188 circular autocorrelation technique (Supplementary Fig. 1). Suppose the length of the data

189 pool is $N$, the length of autocorrelation function should also be $N$. The tail of the pool was

190 linked to the head. In this way, when the pool was full, the algorithm turns to the head of

191 the data pool to restore and retrieve the new data. To compute the amplitude of the

192 autocorrelation function, we integrated one cycle of data.

193 (4) Computation of $R^{\prime}$ and $\mathrm{SpO}_{2}$ according to Eqs. (9)-(12). Every 6 seconds a new set 194 of results were generated.

\subsection{Data analysis}

196 The new algorithm--autocorrelation modeling of $\mathrm{SpO}_{2}$--was carried out on Matlab

197 (MathWorks, USA). Linear regression and other statistical analysis were done by

198 OriginPro v9.0 (OriginLab, USA) and Excel (Microsoft, USA). Statistical data were

199 represented as mean $\pm \mathrm{SD}$. 


\section{Results}

\subsection{Autocorrelation modeling results}

202 The new autocorrelation modeling method was built based on two linearity assumptions:

203 1) the amplitude of original AC component is proportional to square root of that of 204 autocorrelation function as denoted by Eq. (9), and 2) the substitution of $R$ with $R$ ' in Eq.

205 (2). Here, we test the two linearity assumptions.

206 First, we created PPG signals for $\mathrm{PI}=3 \%, \mathrm{PR}=75$, and $\mathrm{SpO}_{2}(\%)$ ranges from 36 to 100 207 using Fluke Index. The simulated signal at each $\mathrm{SpO}_{2}$ was recorded and transmitted to 208 PC.

209 We calculated the magnitudes of original PPG AC component $\left(P_{A C}\right)$ and those of the 210 autocorrelation functions $\left(P^{\prime}{ }_{A C}\right)$. Since values of $P_{A C}$ under different $\mathrm{SpO}_{2}$ settings are 211 different, a sweep of $\mathrm{SpO}_{2}(\%)$ from 36 to 100 yielded a sufficient multi-level coverage of 212 PPG signals. At each $\mathrm{SpO}_{2}$ settings, we calculated $P_{A C}$ and $P^{\prime}{ }_{A C} 10$ times. Linear 213 regression was conducted on means of each pair $(n=10)$. It showed a nearly perfect 214 linearity between $P_{A C}$ and $\left(P_{A C}^{\prime}\right)^{1 / 2}$ (Pearson's r=0.99967, Fig. 4a). Further, the amplitude 215 transfer factor $k$ in Eq. (9) can be calculated from the slope of the regression line. In this 216 case, the wavelength was $\lambda_{1}$ and $k_{1}=1 / 0.348=2.874$.

217 After computation of $k_{1}, k_{2}, P^{\prime}{ }_{A C 1}, P^{\prime}{ }_{A C 2}, P_{D C 1}$ and $P_{D C 2}$, we calculated $R$ and $R^{\prime}$ 218 according to Eqs. (10) and (11). Linear regression of means of $R^{\prime}$ on those of $R(n=10)$ 219 yielded a regression line with slope of 1.004 and Pearson's r of 0.9998 (Fig. 4b).

\subsection{Accuracy}

221 To test $\mathrm{SpO}_{2}$ extraction accuracy of the autocorrelation modeling method, we modeled the 222 low perfusion scene by simulating PPG signals with small PI (say, $\mathrm{PI}=0.2 \%$ and $0.1 \%$ ) 223 (Fig. 5). A sweep of $\mathrm{SpO}_{2}(\%)$ from 64 to 96 was generated by Fluke Index. In PI=0.2\% 
cases, all the $\mathrm{SpO}_{2}$ were accurately estimated with a high precision $(\mathrm{SD} / \mathrm{mean}<3 \%)$ (Fig.

6a, Table 1). In $\mathrm{PI}=0.1 \%$ cases, $\mathrm{SpO}_{2}$ estimates were also accurate $(\mathrm{SD} / \mathrm{mean}<5 \%)$ (Fig.

226 6b, Table 1).

\subsection{Stability}

228 To test the stability of the autocorrelation modeling method, we continuously recorded the

229 simulated PPG signal $\left(\mathrm{PI}=0.2 \%, \mathrm{PR}=70, \mathrm{SpO}_{2}=94 \%\right)$ for 1 hour. The mean $\mathrm{SpO}_{2}$ fall

230 between 92 and $96(94.3 \pm 0.6)$ (Fig. 6c). Further, we calculated PR from the

231 autocorrelation function and got a stable and accurate estimation (69.99 \pm 0.04$)$ (Fig. 6d).

\section{$232 \quad 3.4$ Motion-tolerance}

233 To test the motion-tolerance of the autocorrelation modeling technique, we recorded the

234 PPG signals of our fingers with and without shaking with our home-made dual-

235 wavelength sensor ${ }^{[13]}$. The experiment recorded two finger shaking (about 1 to 4 seconds

236 and 9 to 11 seconds, Fig. 7a). Apparently, the PPG signals with finger shaking were

237 contaminated by interference (Fig. 7a). We calculated the autocorrelation function results

238 of the PPG signals contaminated by interference. The interference was severely

239 suppressed (Fig. 7b).

\section{Discussion}

241 In this paper, we have described a new $\mathrm{SpO}_{2}$ extraction method called autocorrelation

242 modeling. It involved computation of the autocorrelation function and relating its

243 magnitude to that of the original PPG signals. Autocorrelation strongly suppressed the

244 noise and picked out the weak PPG signals under low perfusion condition. We

245 demonstrated a good linearity between the magnitude of PPG signals and the square root

246 of that of the autocorrelation function, upon which $\mathrm{SpO}_{2}$ could be accurately extracted. 
247 The validation experiments using $\mathrm{SpO}_{2}$ simulator demonstrated the high-accuracy of the 248 method in case of low perfusion $(\mathrm{PI}=0.2 \%)$. Besides, the application of autocorrelation 249 technique also strengthened the ability of motion-tolerance.

250 In the clinical use of pulse oximetry, the $\mathrm{SpO}_{2}$ determination under low perfusion 251 condition caused by low ambient temperature or cardiogenic shock ${ }^{[2,3]}$ is severely affected 252 by noise. This brings a lot of trouble to clinical work, such as false decreasing of $\mathrm{SpO}_{2}$, 253 making it important to improve the accuracy of $\mathrm{SpO}_{2}$. Compared with the adaptive 254 method using extra hardware, like accelerators to sense the motion, the new method 255 enhances PPG signals by using its inherent cyclic properties. Since the computation of 256 autocorrelation function could be achieved by Fast Fourier Transformation (FFT), the new 257 method is suitable for real-time application with little computation and high efficiency. In 258 the future, the method needs to be further improved to adapt to the accurate measurement 259 under the condition of lower perfusion(PI=0.1\%) and lower SNR, especially under the 260 condition of large motion interference.

\section{Conclusions}

262 We introduced a method named autocorrelation modeling to solve the issue of inaccurate $263 \mathrm{SpO}_{2}$ measurement under low perfusion in the clinical use. The validation experiments 264 showed the autocorrelation modeling method can strongly suppress the noise and pick out 265 the weak PPG signals under low perfusion condition. The autocorrelation modeling 266 technique showed a high accuracy of the method in case of low perfusion $(\mathrm{PI}=0.2 \%)$, and 267 also strengthened the ability of motion-tolerance. The validation experiments showed a strong potential in real-time clinical use for the autocorrelation modeling method. 


\section{Declarations}

273 Ethics approval and consent to participate:

274 All authors and participants declare that we have read and have abided by the statement of ethical standards

275 Consent for publication:

276 None of the material related to this manuscript has been published or is under consideration for publication

277 elsewhere, including the internet. All authors and participants understand that the information will be

278 published, have read this manuscript and approve to have it considered exclusively for publication in BMC

279 Medical Informatics and Decision Making.

280 Availability of data and materials:

281 All data generated and analysed during this study are included in this published article, and any further

282 details of this study are available from the corresponding author on reasonable request.

283 Competing interests:

284 All authors declare that we have no competing interests.

\section{Funding:}

286 This work was financially supported by Shenzhen Med-Link Electronics Tech Co., Ltd.

287 Authors' contributions:

288 Tan Shuangping designed the method described in this manuscript and designed the experiment to verify

289 the method, and was the major contributor in writing the manuscript. Wei Jie and Chen Hao analyzed and

290 interpreted the experiment data, and also contributed to the writing of the manuscript. Zhang Tong, Deng

291 Youfeng and Zuo Hongbin provided a lot of help for the experimental method and writing of this paper. Wu

292 Xiali analyzed and interpreted the experiment data. All authors read and approved the final manuscript.

293 Acknowledgements:

294 Not applicable.

295 Author details:

296 1. The Jiangxia District No.1 People's Hospital of Wuhan, Wuhan, 430200, China

297 2. State Key Laboratory of Information Engineering in Surveying, Mapping and Remote Sensing, Wuhan

298 University, Wuhan, 430079, China

\section{$300 \quad$ References}

301 1. Docherty, Brendan. Cardiorespiratory physical assessment for the acutely ill: 2. British Journal of

302 Nursing. 2002;11: 800-807.

303 2. Liu Guangda, Guo Wei, Zhu Ping, Bai Mingming. Blood oxygen saturation measurement based on

304 volume signal analysis. LASER \& INFRARED. 2009;02: 169-172.

305 3. Zhang Kun, JiaoTeng, Fu Feng, ZhangWen, Dong Xiuzhen. Motion artifact cancellation in

306 photoplethysmography using reconstruction of wavelet transform modulus maxima. Chinese Journal of

307 Scientific Instrument. 2009;3:586-589. 
308 4. Wen Daxiang, Zhou Yin, Wang Shanjuan, Hang Yannan. The effects of motion and low perfusion on the

309 accuracy of pulse oximetry. J Clin Anesthesiol. 2004;06: 333-335.

310 5. Mohamed KD. System and methods for determing blood oxygen saturation values using complex number 311 encoding. US Patent: US7440787B2, Oct.21,2008.

312 6. Foo JYA, Wilson SJ. A computational system to optimise noise rejection in photoplethysmography

313 singals during motion or poor perfusion states. Med Biol Eng Comput. 2006;44:140-145.

314 7. Yousefi R, Nourani M, Ostadabbas S, Panahi I. A motion-tolerant adaptive algorithm for wearable

315 photoplethysmographic biosensors. IEEE J Biomed Health Inform. 2014;2:670-681.

316 8. Byung SK, Sun KY. Motion artifact reduction in photoplethysmography using independent component

317 analysis[J]. IEEE Transactions on Biomedical Engineering. 2006;3: 566-568.

318 9. Yan YS, Zhang YT. An efficient motion-resistant method for wearable pulse oximeter. IEEE T Inf

319 Technol B. 2008;12:399-405.

320 10. Yan Xinzhong, Yang Jing, Guo Lue. Study on measuring method of human oxygen saturation, medical 321 equipment. 2005;12: 1-4.

322 11. Chen Mingkui, Liu Zhengping .The detection of weak sinusoidal signal based on multi-layer

323 autocorrelation. Light Industry Machinery. 2006;3:112-115.

324 12. Fan Xiaozhi, Zhao Lizhi, Huang Xiaohong. An inspecting technology for weak sinusoidal signal based 325 on multi -layer autocorrelation. Journal of Chinese Computer Sy stems. 2007;3: 566-568.

326 13. Tan S.P., Ai Z.G., Yang Y.X., Xie Q.G.. Design of a pulse oximeter used to low perfusion and low 327 oxygen saturation. Chinese Jounal of Medical Instrumentation. 2013;37:189-196.

Figures: 

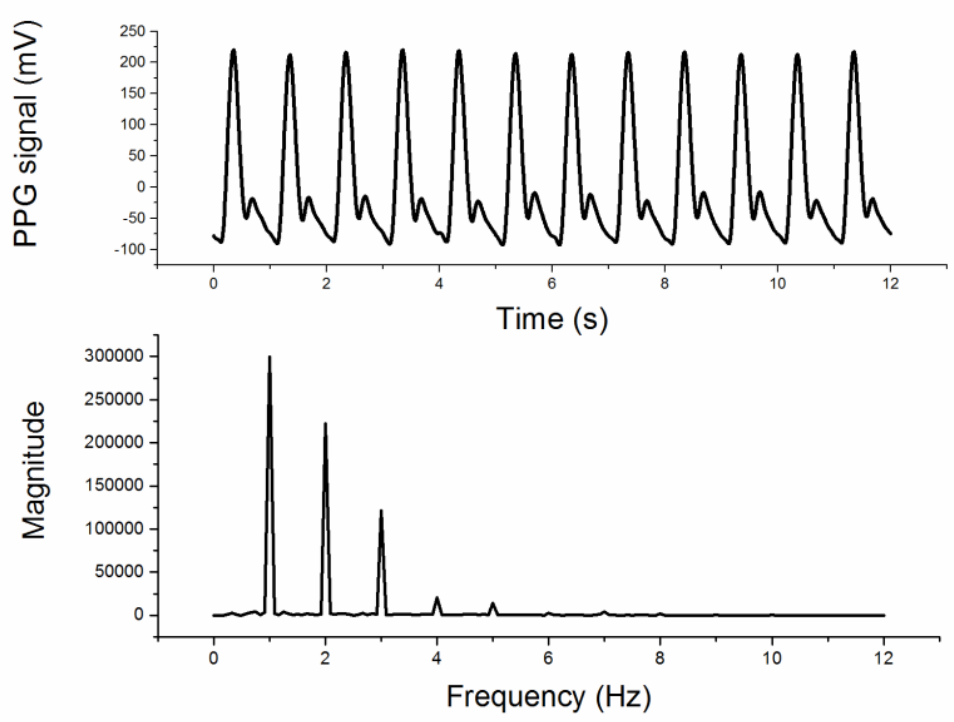

(a)
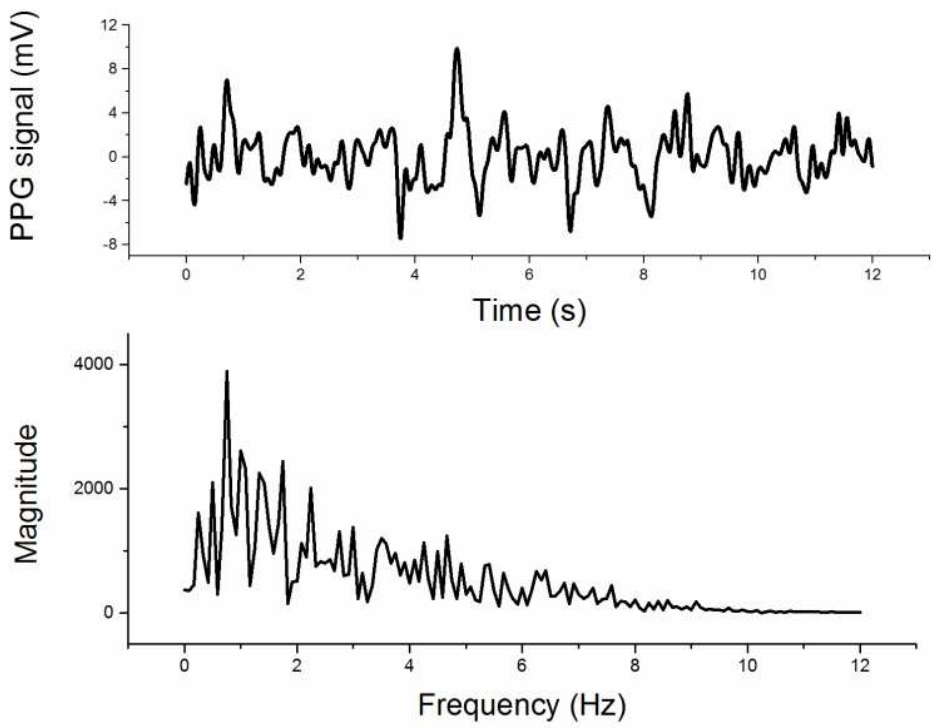

(b)

335 Fig. 1 Simulated PPG signal and its Fourier spectra. (a) Normal case: $\mathrm{PI}=3 \%, \mathrm{SpO}_{2}=96 \%, \mathrm{PR}=60$ times $/ \mathrm{min}$. 


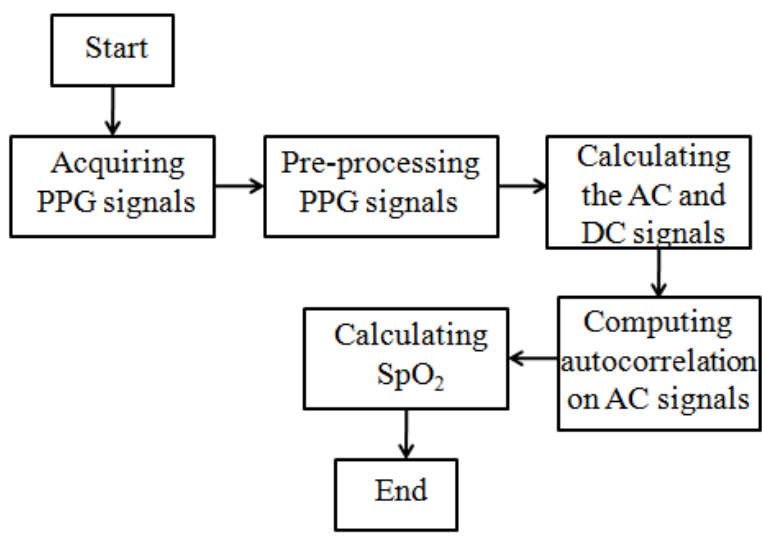

Fig. 2 Overall process of autocorrelation modeling method

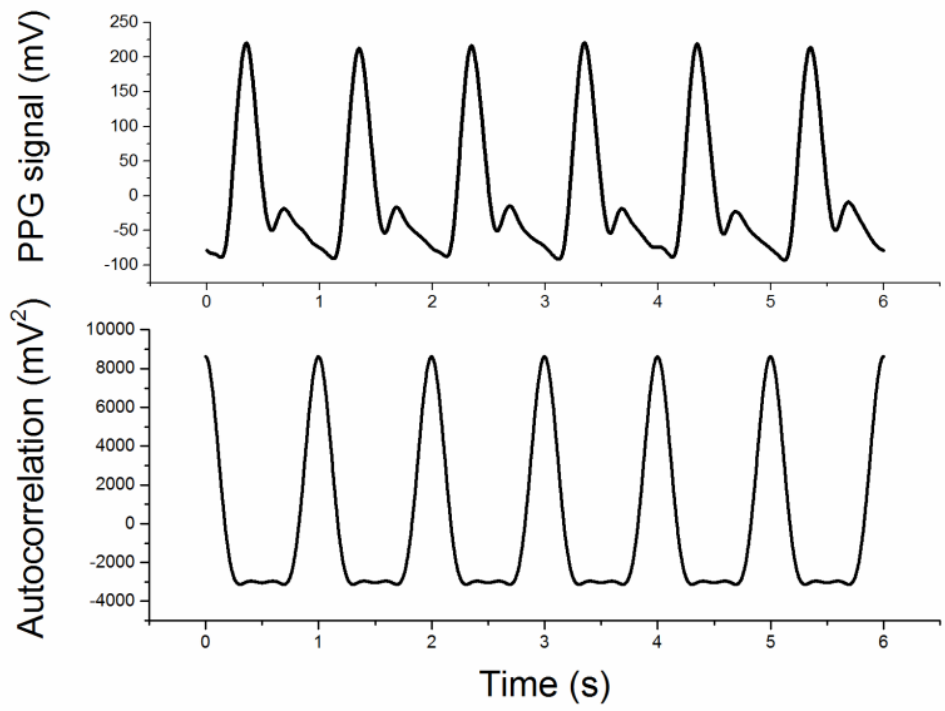




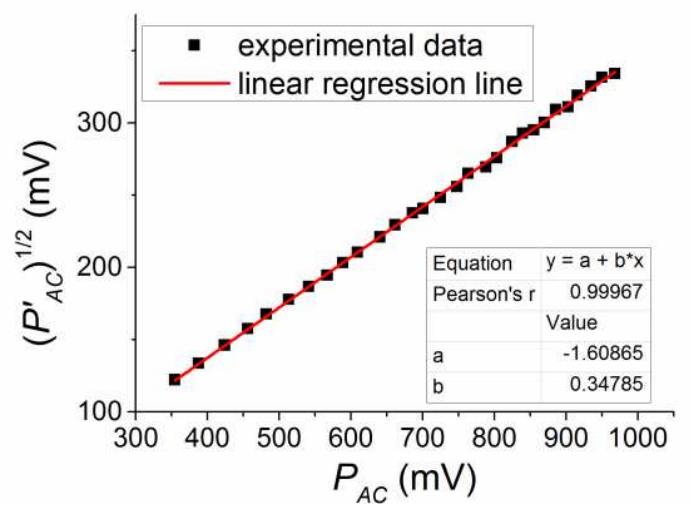

(a)

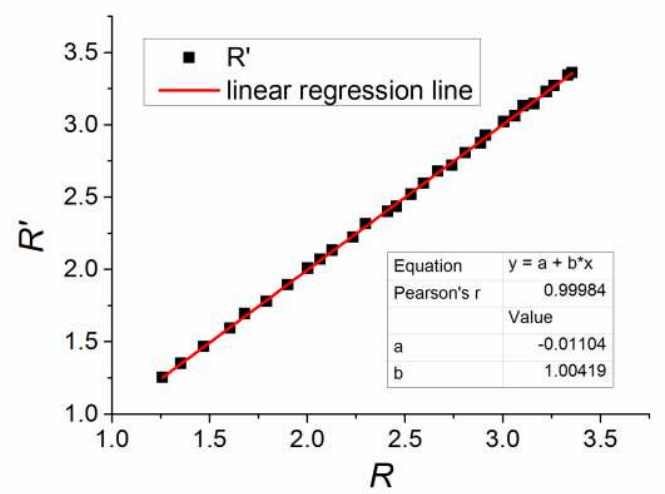

(b)

351 Fig. 4 Linear regression of the critical intermediate parameters. (a) Linear regression of square root of the

352 amplitude of autocorrelation function of PPG AC component on original PPG AC amplitude; (b) Linear 353 regression of $R^{\prime}$ on $R$ 

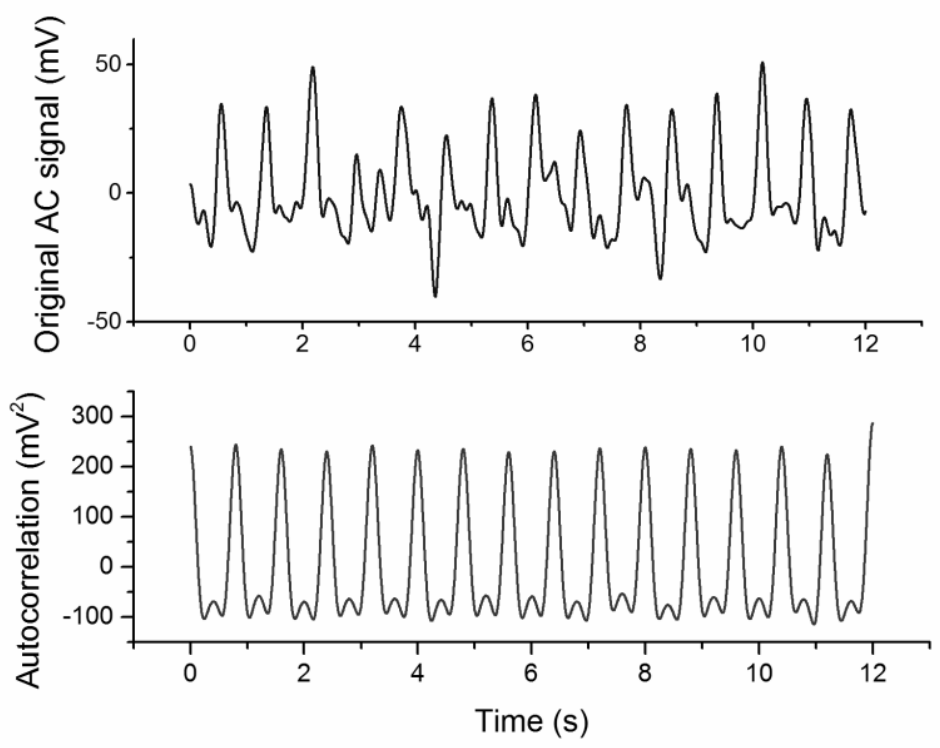

(a)
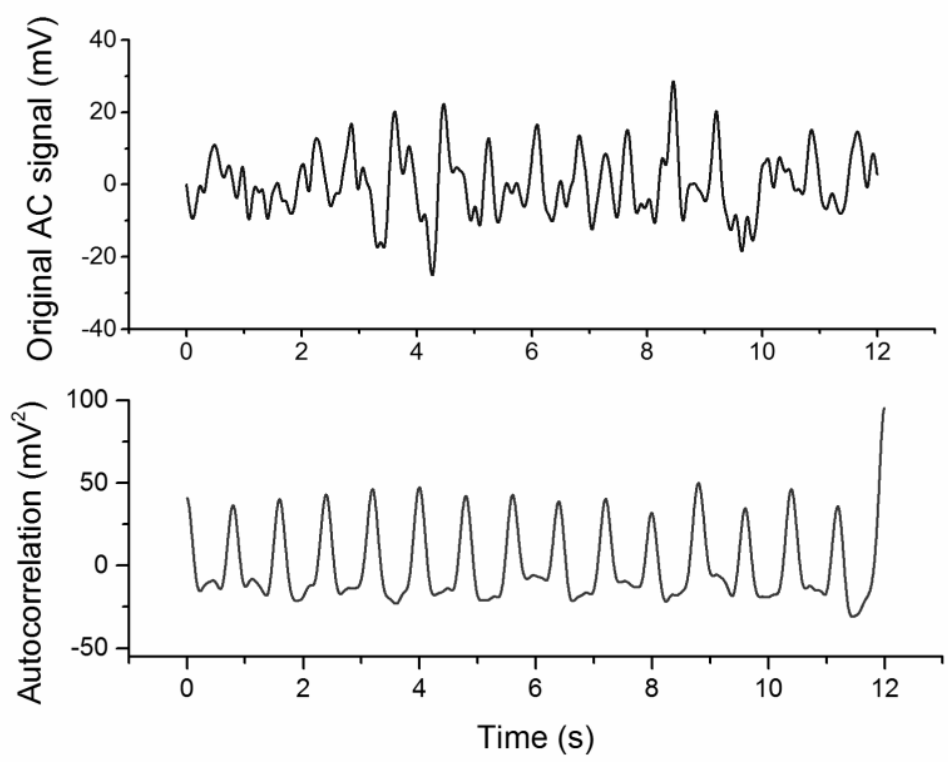

(b)

361 Fig. 5 Simulated PPG AC signal under low perfusion condition and its autocorrelation function. (a) $362 \quad \mathrm{PI}=0.2 \%$; (b) $\mathrm{PI}=0.1 \%$ 


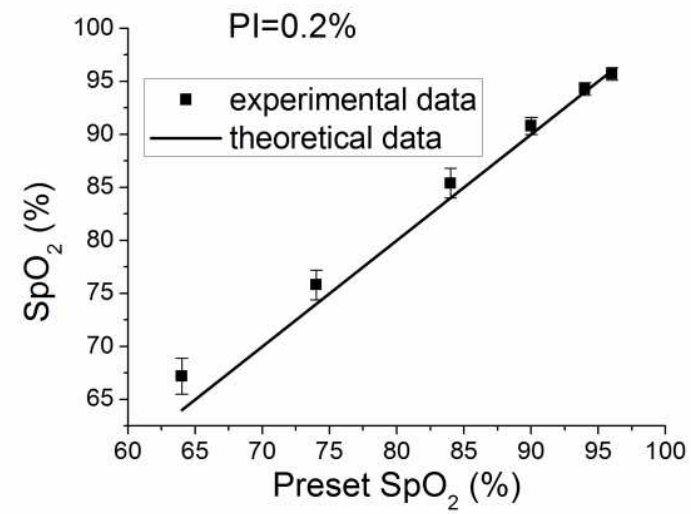

(a)

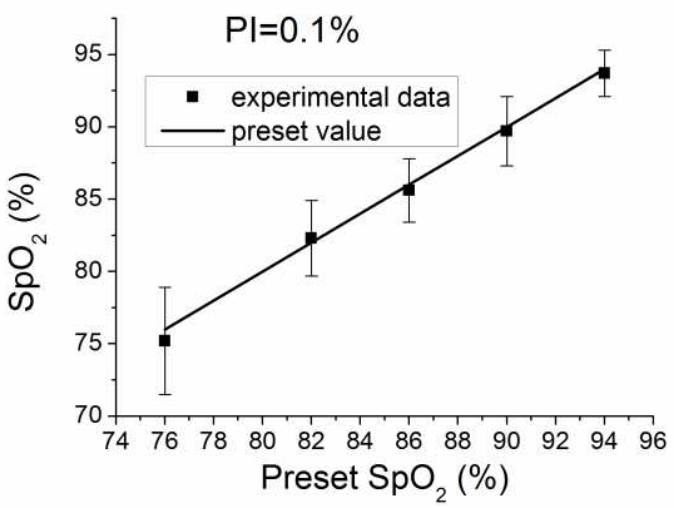

(b)

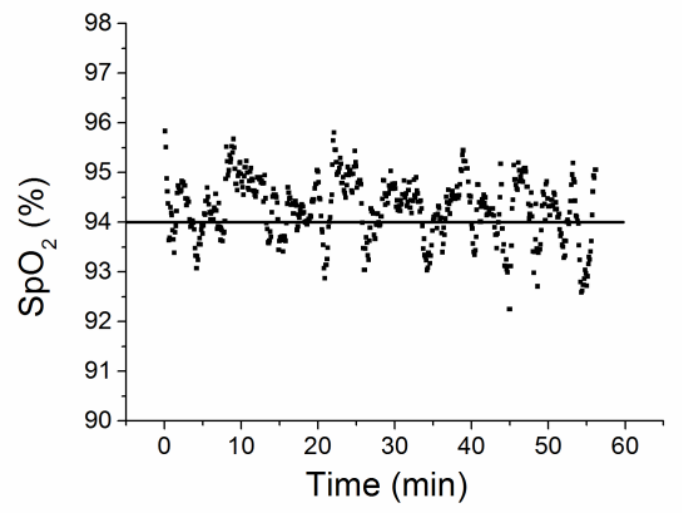

(c) 


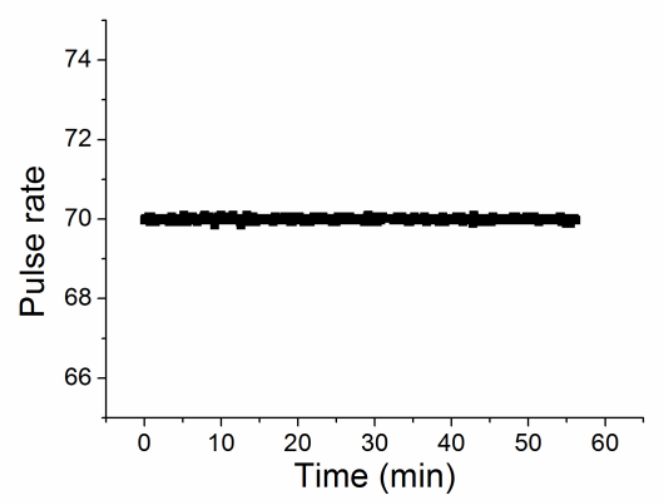

(d)

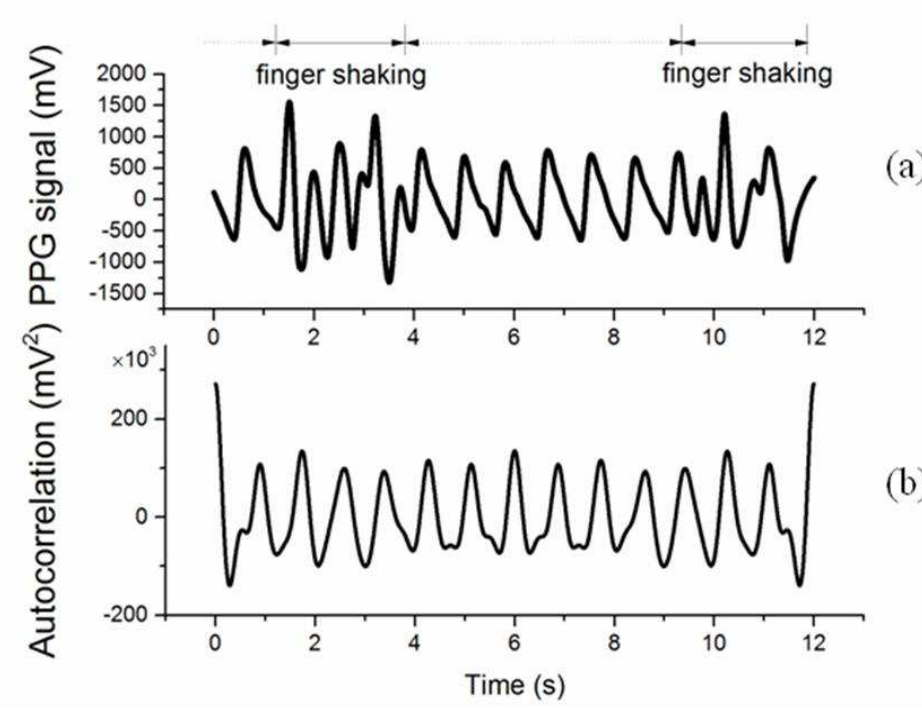


Conventional linear autocorrelation

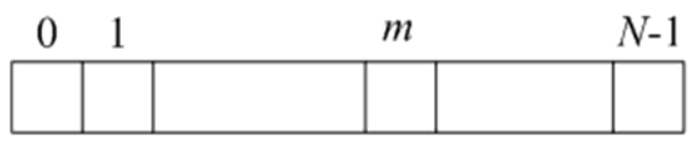

(a)
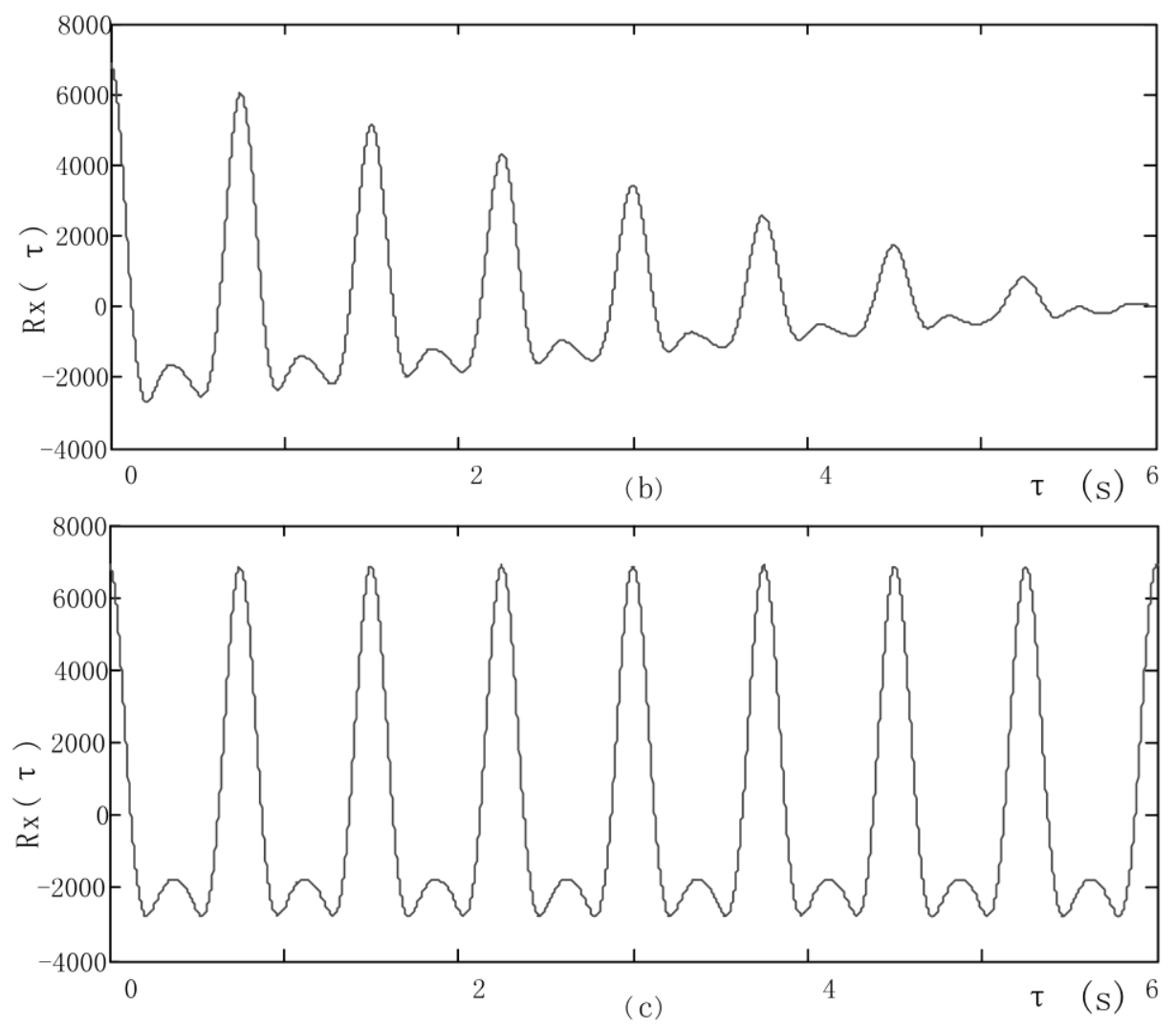

Supplementary fig. 1 (a) Memory management of conventional linear autocorrelation and circular autocorrelation computation. (b) Autocorrelation function of a typical PPG signal calculated by conventional linear autocorrelation. (c) Autocorrelation function calculated by circular autocorrelation 
389 Tables:

390 Table $1 \mathrm{SpO}_{2}$ estimates under low perfusion condition

\begin{tabular}{|c|c|c|c|c|c|c|c|c|c|c|c|}
\hline \multirow[b]{2}{*}{ Preset $\mathrm{SpO}_{2}(\%)$} & \multicolumn{6}{|c|}{$\mathrm{PI}=0.2 \%$} & \multicolumn{5}{|c|}{$\mathrm{PI}=0.1 \%$} \\
\hline & 64 & 74 & 84 & 90 & 94 & 96 & 76 & 82 & 86 & 90 & 94 \\
\hline Mean of estimates & 67.2 & 75.8 & 85.4 & 90.8 & 94.3 & 95.7 & 75.2 & 82.3 & 85.6 & 89.7 & 93.7 \\
\hline SD of estimates & 1.7 & 1.4 & 1.4 & 0.8 & 0.6 & 0.6 & 3.7 & 2.6 & 2.2 & 2.4 & 1.6 \\
\hline
\end{tabular}

391 
Figures
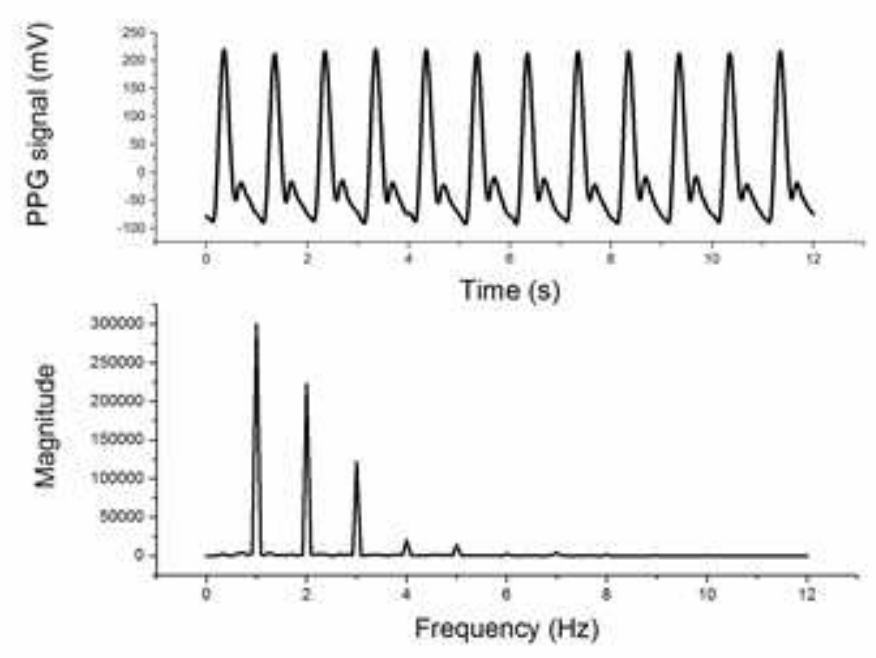

(a)
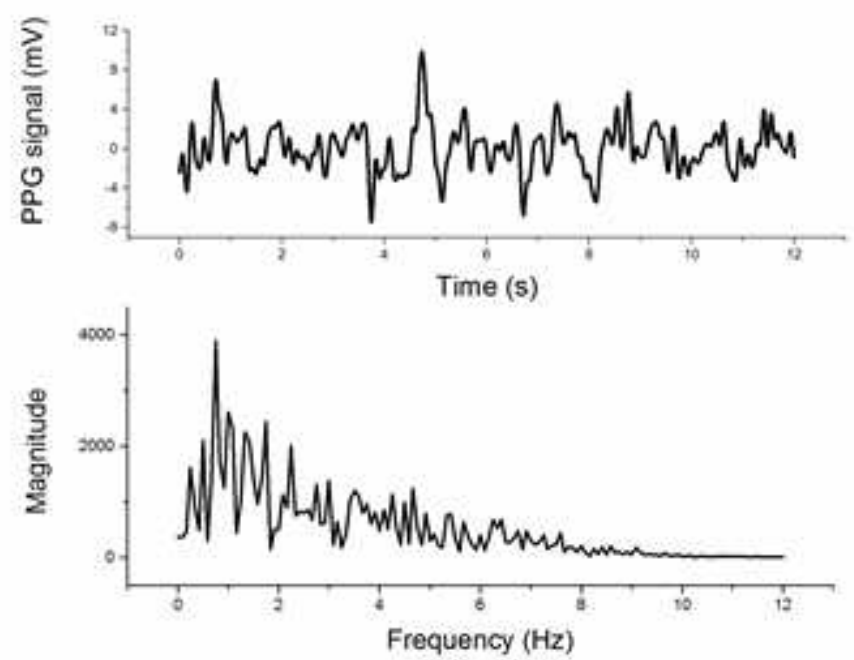

(b)

\section{Figure 1}

Simulated PPG signal and its Fourier spectra. (a) Normal case: $\mathrm{PI}=3 \%, \mathrm{SpO}=96 \%, \mathrm{PR}=60$ times $/ \mathrm{min}$. (b) Low perfusion case: $\mathrm{Pl}=0.075 \%, \mathrm{SpO} 2=96 \%, \mathrm{PR}=60$ times $/ \mathrm{min}$ 


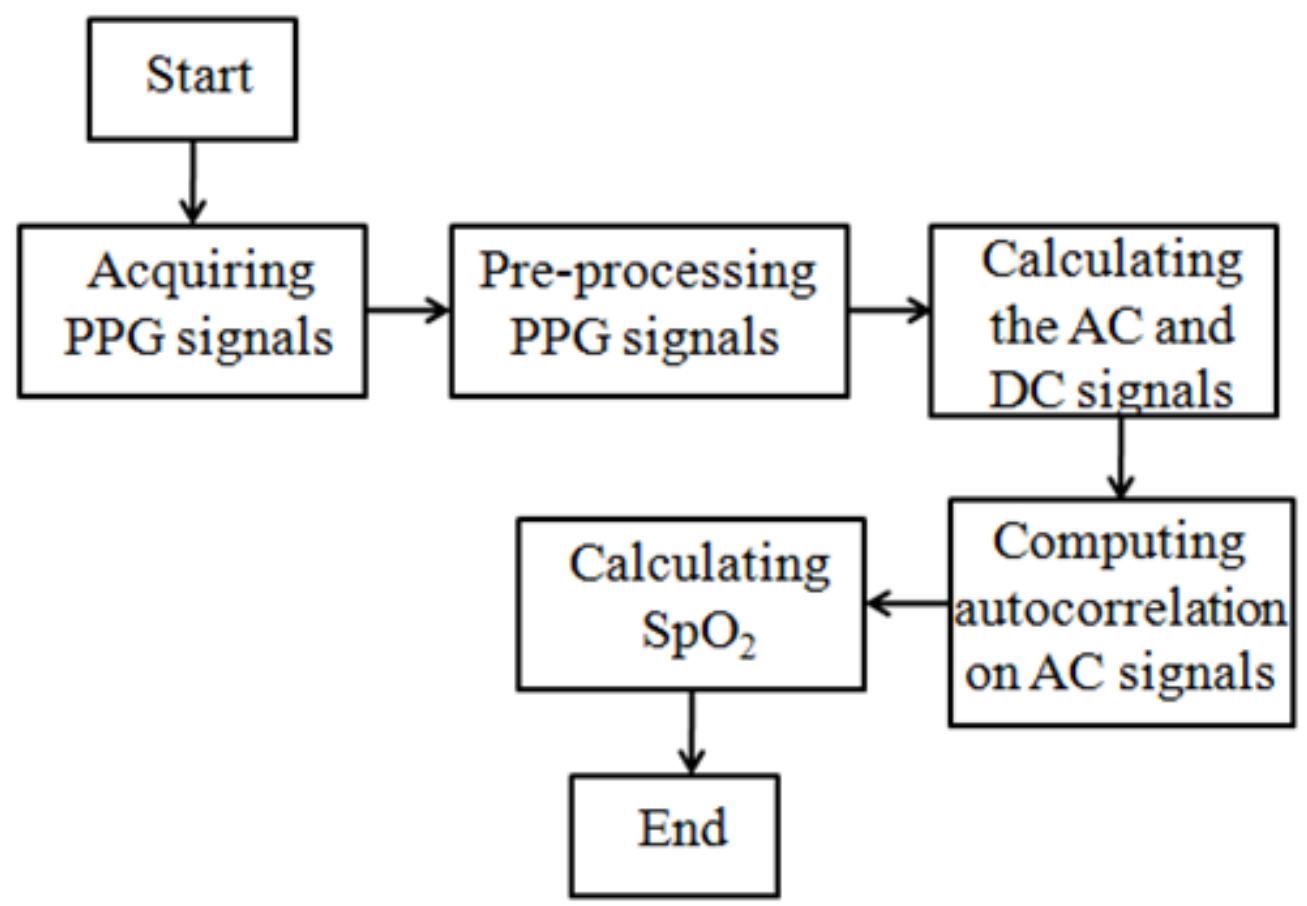

Figure 2

Overall process of autocorrelation modeling method

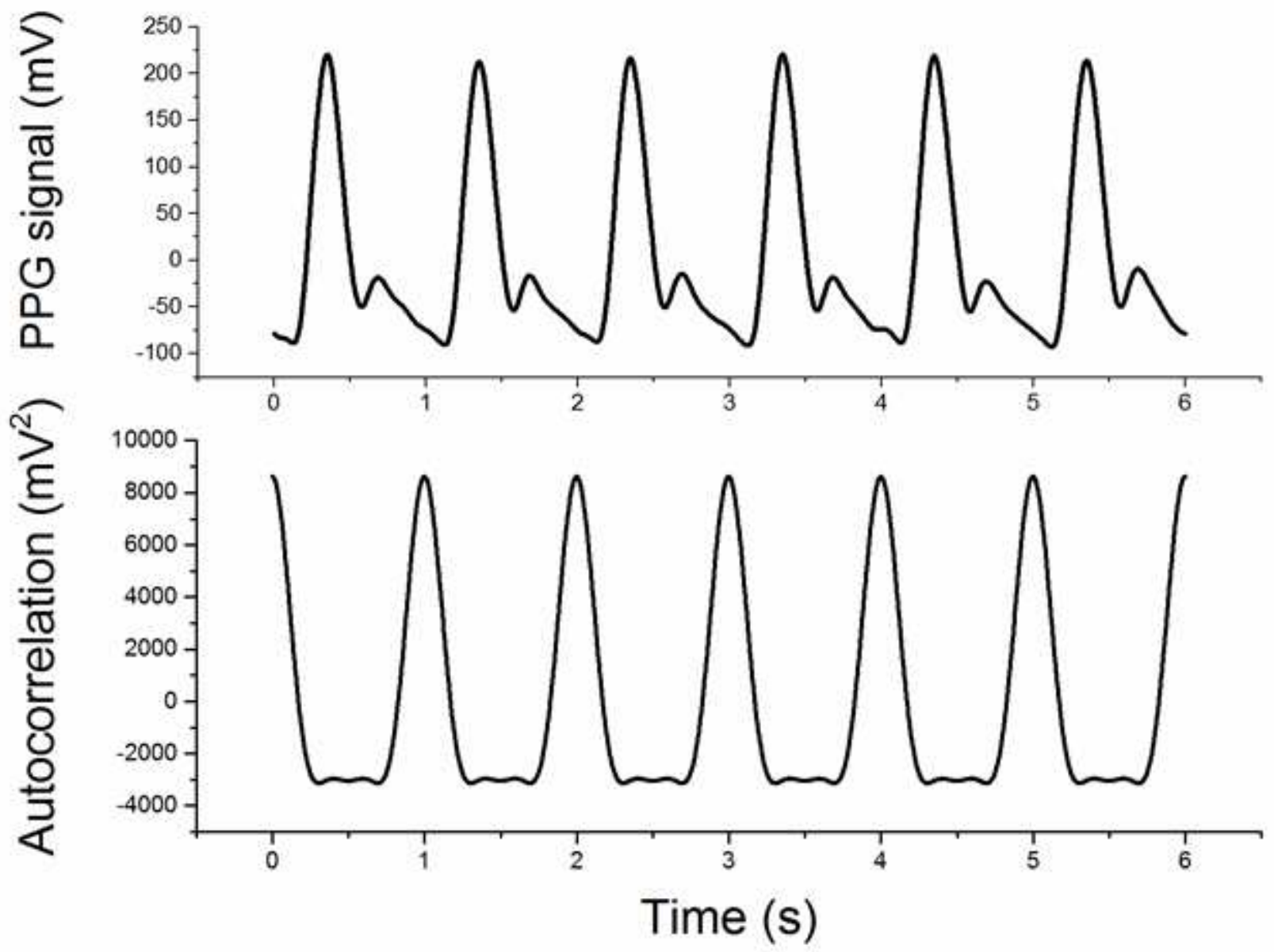


Figure 3

Autocorrelation function of a typical normal PPG signal

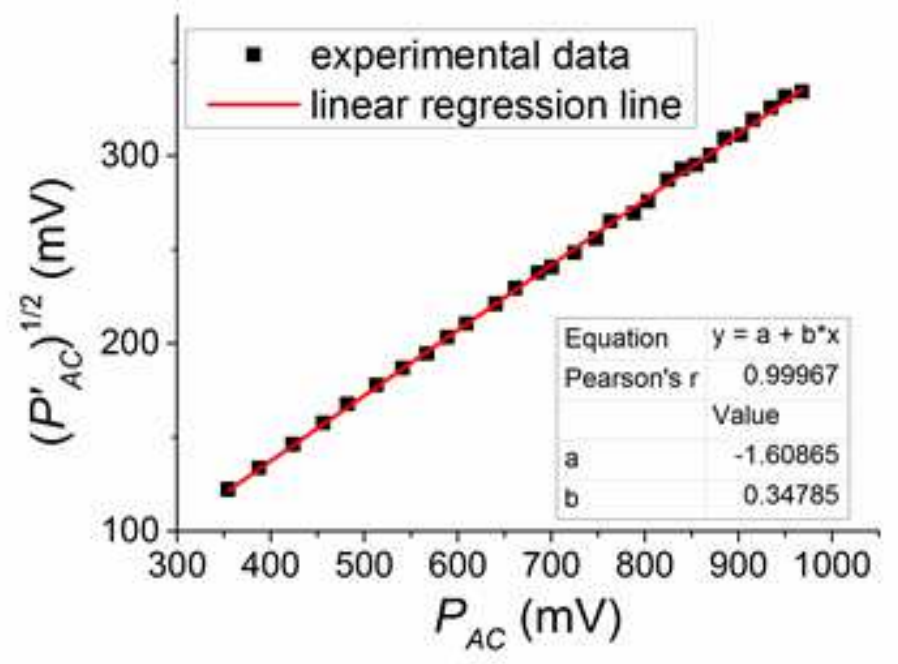

(a)

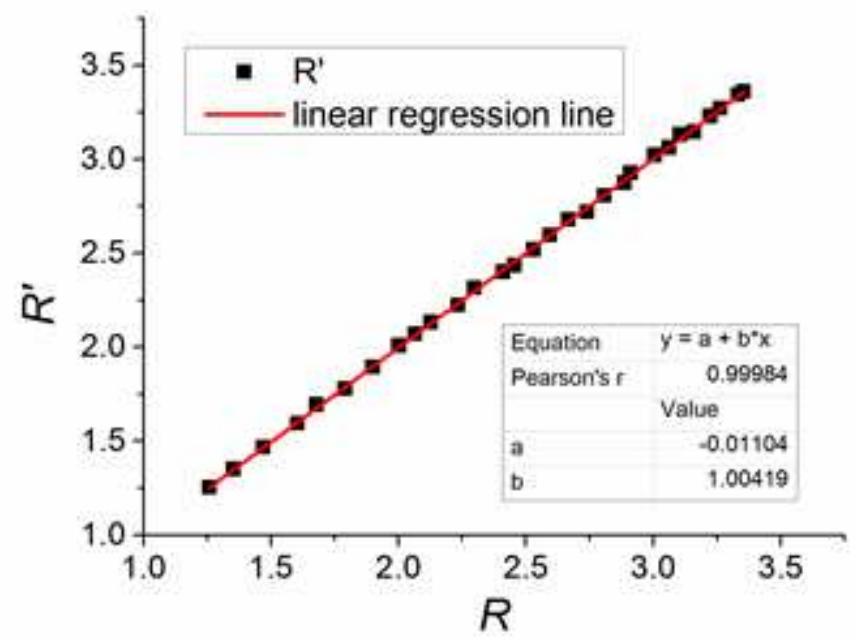

(b)

\section{Figure 4}

Linear regression of the critical intermediate parameters. (a) Linear regression of square root of the amplitude of autocorrelation function of PPG AC component on original PPG AC amplitude; (b) Linear regression of $\mathrm{R}^{\prime}$ on $\mathrm{R}$ 

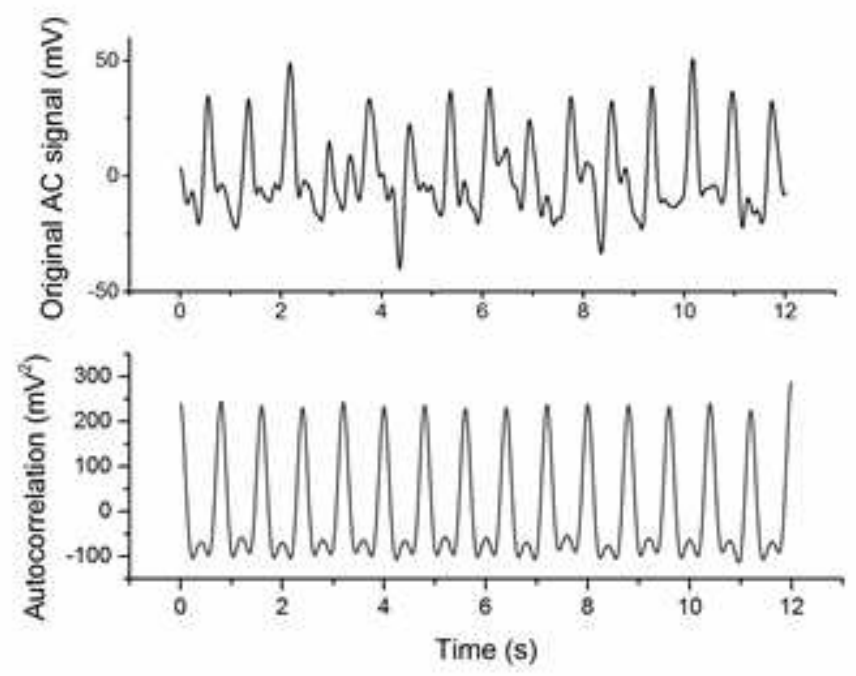

(a)
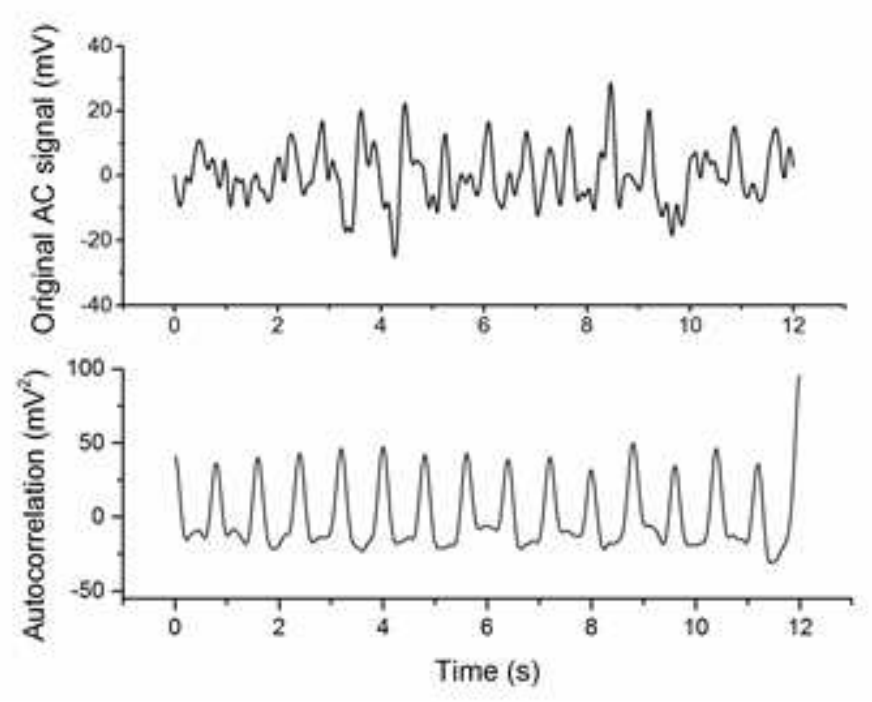

(b)

Figure 5

Simulated PPG AC signal under low perfusion condition and its autocorrelation function. (a) $\mathrm{Pl}=0.2 \%$; (b) $\mathrm{Pl}=0.1 \%$ 


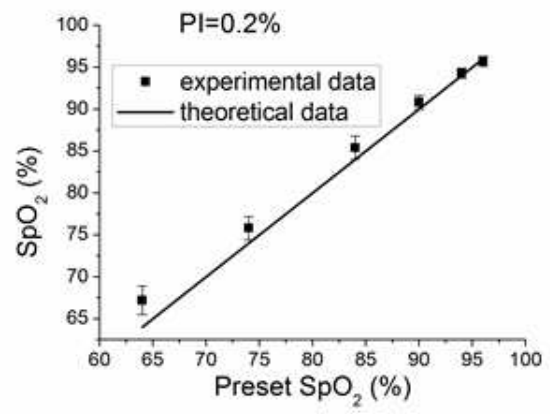

(a)

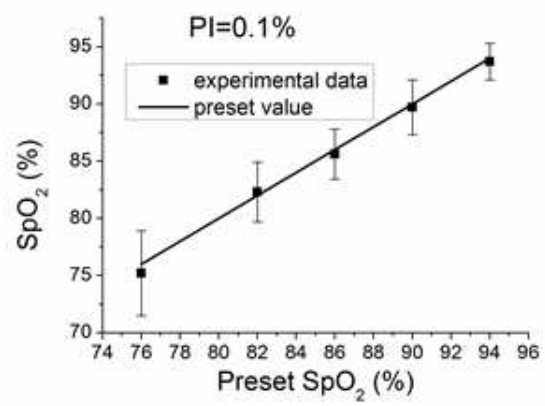

(b)

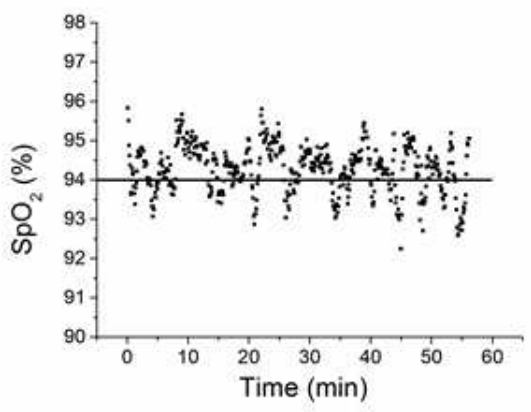

(c)

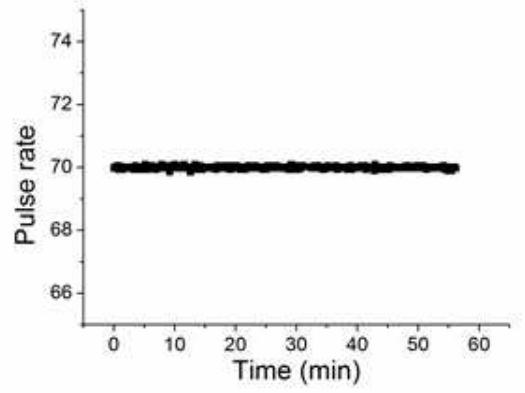

(d)

\section{Figure 6}

SpO2 and PR extracted using autocorrelation modeling method 


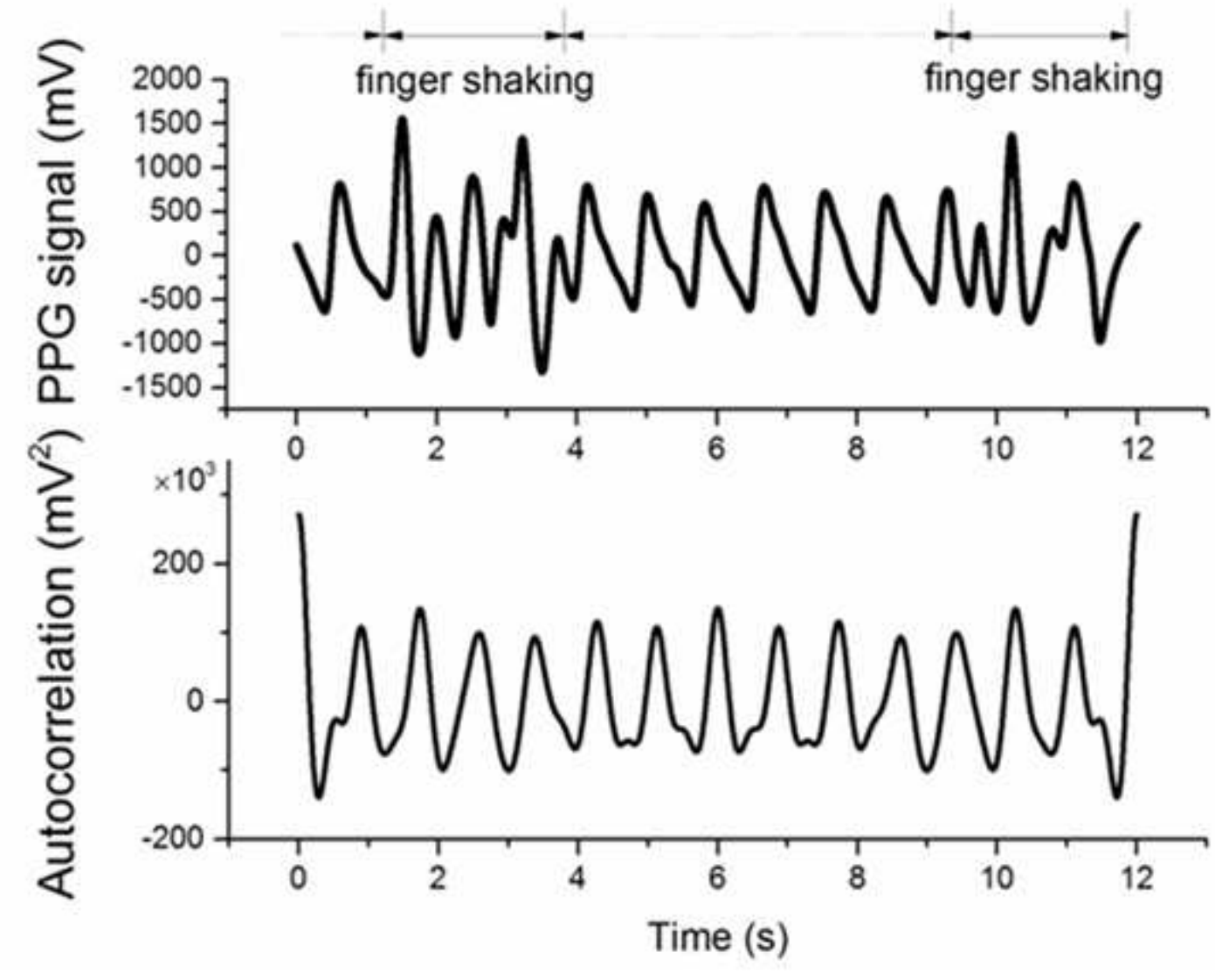

(a)

(b)

Figure 7

Recorded PPG signal in case of body movement

\section{Supplementary Files}

This is a list of supplementary files associated with this preprint. Click to download.

- Supplementary.docx 